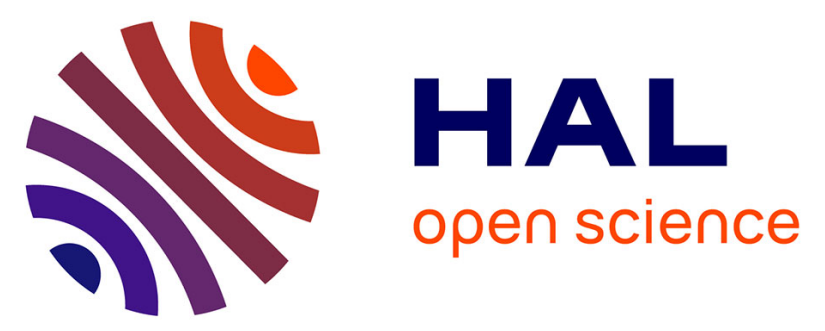

\title{
Effet du niveau d'alimentation énergétique de génisses laitières de races Holstein et Normande jusqu'à l'âge de 14 mois sur les performances durant les périodes d'élevage et de lactation
}

\author{
Jl Troccon, A Muller, Jr Peccatte, M Fargetton
}

\section{- To cite this version:}

Jl Troccon, A Muller, Jr Peccatte, M Fargetton. Effet du niveau d'alimentation énergétique de génisses laitières de races Holstein et Normande jusqu'à l'âge de 14 mois sur les performances durant les périodes d'élevage et de lactation. Annales de zootechnie, 1997, 46 (1), pp.27-41. hal-00889674

\section{HAL Id: hal-00889674 https://hal.science/hal-00889674}

Submitted on 1 Jan 1997

HAL is a multi-disciplinary open access archive for the deposit and dissemination of scientific research documents, whether they are published or not. The documents may come from teaching and research institutions in France or abroad, or from public or private research centers.
L'archive ouverte pluridisciplinaire HAL, est destinée au dépôt et à la diffusion de documents scientifiques de niveau recherche, publiés ou non, émanant des établissements d'enseignement et de recherche français ou étrangers, des laboratoires publics ou privés. 


\title{
Effet du niveau d'alimentation énergétique de génisses laitières de races Holstein et Normande jusqu'à l'âge de 14 mois sur les performances durant les périodes d'élevage et de lactation
}

\author{
$\mathrm{JL}_{\text {Troccon }}{ }^{1 *}$, A Muller ${ }^{2}$, JR Peccatte ${ }^{2}$, M Fargetton ${ }^{3}$ \\ I Station de recherches sur la vache laitière, Inra, 35590 Saint-Gilles; \\ ${ }^{2}$ Domaine d'expérimentation du Pin au Haras, Inra, 61310 Exmes ; \\ ${ }_{3}^{3}$ Domaine d'expérimentation, Inra, 93310 Petit-Bourg, France
}

(Reçu le ler septembre 1995 ; accepté le 22 août 1996)

\begin{abstract}
Summary - Energetic feeding from birth to 14 months of Holstein and Norman dairy heifers and next performances. Holstein $(n=71)$ and Norman $(n=32)$ heifers born during 3 successive years were allocated to two Moderate $(\mathrm{M})$ and High $(\mathrm{H})$ energetic treatments from birth to the mean age of $14( \pm 1)$ months. Feed restriction in milk, concentrate and corn silage was equal to 400 UFL. Live weight gains were 691 and $845 \mathrm{~g} /$ day. Live weight was decreased by $17 \%$ with the $M$ treatment ( $335 \mathrm{vs} 404 \mathrm{~kg}$ ). From the end of the experimental period to the first calving, the $\mathrm{M}$ heifers realized a compensatory growth compared to the $\mathrm{H}$ heifers $(+95 \mathrm{~g} /$ day), which was slower in Holstein heifers than in Norman heifers. The fertility of the Holstein heifers was higher with the M treatment than with the $\mathrm{H}$ treatment $(+24 \%$ after two artificial inseminations, $P<0.02$ ). The fertility of the Norman heifers did not differ between the $\mathrm{M}$ and $\mathrm{H}$ treatments. However, milk production was not affected by the treatments. Cow fertility was high when Holstein and Norman heifers were fed the $\mathrm{H}$ and $\mathrm{M}$ treatments, respectively, probably because they have high (Holstein) and low (Norman) levels of milk production. Health problems, culling and meat production were similar with the two treatments. Thus, late calving heifers may receive limited energetic levels at an early age. Nevertheless, Norman heifers have higher compensatory growth than Holstein heifers.
\end{abstract}

dairy heifers / feeding / energy / milk / longevity

\footnotetext{
* Correspondance et tirés à part.

Fax : (33) 0299285101 ; courriel : troccon@st-gilles.rennes.inra.fr
} 
Résumé - Des génisses de races Holstein $(n=71)$ et Normande $(n=32)$ nées au cours de 3 années successives ont été soumises à deux traitements énergétiques, Modéré $(\mathbf{M})$ et Haut $(\mathrm{H})$, entre les âges de 6 semaines et 14 mois. Au cours de la période expérimentale, les apports énergétiques en lait entier, en aliment concentré et en ensilage de maïs du traitement M ont été de 400 UFL inférieurs à ceux du traitement $\mathrm{H}$. À la fin de la période expérimentale, le poids vif des génisses $\mathrm{M}$ a été de $17 \%$ plus faible ( 335 contre $404 \mathrm{~kg}$ ). Les gains de poids vif des génisses $\mathrm{M}$ et $\mathrm{H}$ ont été respectivement de 691 et $845 \mathrm{~g} /$ jour. De la fin de la période expérimentale au premier vêlage à l'âge de 3 ans, les génisses $\mathrm{M}$ ont réalisé une croissance compensatrice par rapport aux génisses $\mathrm{H}$ ( $+95 \mathrm{~g} / \mathrm{jour}$ ), plus lente en race Holstein qu'en race Normande. La fertilité des génisses $M$ a été améliorée par rapport aux génisses $\mathrm{H}(+24 \%$ après deux inséminations, $p<0,02)$. En revanche, les productions laitières n'ont pas été affectées par les traitements. La fertilité des vaches Holstein $\mathbf{H}$ et des vaches Normandes $\mathrm{M}$ a été meilleure, sans doute influencée par les productions laitières élevée en race Holstein et faible en race Normande. Les problèmes sanitaires, les causes de réforme et les poids des carcasses des vaches ont été identiques dans les deux traitements. Ainsi, les génisses destinées à un vêlage tardif vers 3 ans peuvent recevoir des apports énergétiques limités dans le jeune âge. Cependant, les génisses Normandes sont plus aptes que les génisses Holstein à réaliser une forte croissance compensatrice.

\section{génisses laitières / alimentation / énergie / lait / longévité}

\section{INTRODUCTION}

La première année d'élevage des génisses laitières est marquée par le passage de l'état de monogastrique à celui de ruminant (3-4 premiers mois) et par l'acquisition de la maturité sexuelle, vers l'âge de 10 mois dans la race Holstein dans des conditions normales de conduite (Troccon et Petit, 1989).

Au cours de la période prépubère, le développement des tissus osseux et musculaire est rapide (Robelin et al, 1986). En revanche, les apports énergétiques prépubères excessifs risquent de réduire le développement du tissu sécréteur mammaire (Jammes et Djiane, 1988) et la production laitière ultérieure (Foldager et Sejrsen, 1991 ; Sejrsen, 1994).

Les génisses destinées à des vêlages précoces vers l'âge de 2 ans nécessitent cependant des croîts assez élevés dans le jeune âge (de la naissance à 6 mois) et un développement rapide et suffisant jusqu'au premier vêlage pour en faciliter le déroulement. Les objectifs suivants ont été proposés dans les races de grand format $(675 \mathrm{~kg}$ de poids adulte) : $200 \mathrm{~kg}$ à l'âge de 6 mois,
$400 \mathrm{~kg}$ à la première insémination et $600 \mathrm{~kg}$ avant le premier vêlage à 24 mois (Troccon, 1993).

Dans le cas de vêlages tardifs (vers l'âge de 3 ans), les croissances peuvent être beaucoup plus modérées, ce qui permettra de réduire le coût de l'alimentation dans le jeune âge (lait, aliment concentré et foin ou ensilage). L'incidence d'une réduction des apports alimentaires de la naissance à l'âge d'environ 15 mois, en vue d'un vêlage tardif à 3 ans, a été étudiée dans ce but au domaine Inra du Pin au Haras.

\section{MATÉRIEL ET MÉTHODES}

\section{Schéma expérimental et traitements}

Les génisses ont été conduites en deux lots séparés destinés à recevoir un régime alimentaire soit Modéré (lot $\mathrm{M}$ ) soit Haut (lot $\mathrm{H}$ ) de la naissance jusqu'à l'âge de 14-15 mois. À partir de la première sortie en pâture vers 14-15 mois, les génisses des deux lots ont été mélangées et ont reçu les mêmes régimes. 
Nos objectifs ont été pour le lot $\mathrm{H}$ d'obtenir une croissance comparable à celle visée pour un vêlage précoce ( $400 \mathrm{~kg}$ à l'âge de 15 mois pour des vaches pesant $675 \mathrm{~kg}$ à l'âge adulte ; Troccon, 1993) et pour le lot $\mathrm{M}$, de réduire le poids vif de $20 \%$ (320 kg à l'âge de 15 mois). Les restrictions énergétiques appliquées aux génisses M par rapport aux génisses $\mathrm{H}$ ont porté sur les apports de lait entier (de 1,5 mois à 3 mois d'âge), d'aliment concentré (de 3 à 9 mois) et de fourrage (de 9 à 15 mois). Les conséquences des 2 régimes ont été mesurées sur le développement corporel, la reproduction, la production laitière et la longévité des animaux (génisses puis vaches).

\section{Troupeau et lots de génisses}

L'essai a porté sur 103 génisses de races $\mathrm{Hol}-$ stein $(n=71)$ et Normande $(n=32)$ nées à la date moyenne du 6 février au cours des hivers 1986 à 1988 au sein du troupeau du domaine Inra du Pin au Haras (Orne, France). Dès la naissance et tous les hivers, les génisses ont été logées par groupes de 8 à 10 dans des cases paillées.

La mise en lot est intervenue peu après la naissance. Les critères de mise en lots ont été la race et la date de naissance, le rang de lactation de la mère et l'origine paternelle.

\section{Alimentation de la naissance à 14-15 mois (période expérimentale)}

Séparées de leur mère dès la naissance, les génisses ont reçu du colostrum au seau pendant 6 jours en 2 repas par jour. Jusqu'au sevrage réalisé à 8 semaines (lot $\mathrm{M}$ ) ou 13 semaines (lot $\mathrm{H}$ ), le lait entier a été distribué individuellement en quantité limitée, en 2 repas par jour (au maximum $4 \mathrm{~kg}$ par repas). Les génisses ont disposé d'aliments solides dès la troisième semaine d'âge : un aliment concentré granulé de $5 \mathrm{~mm}$ de diamètre à base de céréales et de tourteau complémentés en minéraux et en vitamines (au maxi- mum $2 \mathrm{~kg}$ par jour) et du foin distribué à volonté L'alimentation des génisses des deux lots n'a pas différé avant l'âge de 6 semaines, qui marque le début du sevrage des génisses $M$.

Le printemps et l'été suivant la naissance (du 15 mai au 18 octobre), les génisses ont reçu de l'herbe coupée (essentiellement du ray-grass anglais) complémentée en minéraux sans (lot M) ou avec (lot H) l'apport de 1,5 kg d'aliment concentré.

Au cours de l'hiver suivant ( 10 à 15 mois d'âge), les génisses ont reçu de l'ensilage (herbe ou maïs) distribué en quantité limitée (lot $\mathbf{M}$ ) ou à volonté (lot $\mathrm{H}$ ). La période d'application des traitements s'est terminée avec la mise à l'herbe des génisses en moyenne le 11 avril de l'année suivant leur naissance.

\section{Alimentation jusqu'au premier vêlage et au cours des lactations}

De l'âge de 14-15 mois jusqu'au premier vêlage, les génisses $M$ et $H$ regroupées ont reçu ensemble les mêmes régimes alimentaires. Au cours des deux périodes estivales suivantes, les génisses ont pâturé des prairies naturelles ou des prairies temporaires à base de ray-grass anglais. Au cours du troisième hiver, elles ont reçu des quantités identiques et limitées d'ensilage d'herbe ou de maïs. Les vaches ont pâturé en été et ont reçu en hiver un régime à base d'ensilage de maïs distribué à volonté. Les vaches ont été complémentées en aliment concentré selon leur production laitière (Hoden et al, 1988).

\section{Mises à la reproduction et conduite}

La mise à la reproduction des génisses et des vaches est intervenue après synchronisation des cycles sexuels (méthode Synchro-Mate B, Pelot et al, 1984). Les inséminations des génisses et des vaches ont débuté en avril de chaque année, peu avant la mise à l'herbe. Un diagnostic précoce de non-gestation, réalisé systématiquement (dosage de la progestérone plasmatique 21 jours 
après l'insémination), a permis une éventuelle nouvelle induction d'œstrus par une injection de prostaglandines. Ensuite, les génisses et les vaches non gestantes ont été inséminées sur les chaleurs naturelles observées. Les événements concernant la reproduction et le déroulement des vêlages ont été enregistrés.

Les génisses ont été traitées préventivement contre les strongles digestives et la douve. Le tarissement des vaches est intervenu 2 mois avant la date présumée du vêlage suivant.

\section{Mesures et contrôles}

Au cours de la période expérimentale, les quantités offertes et refusées de lait entier, d'aliments concentrés et d'ensilages ont été mesurées tous les jours. En revanche, les quantités de foin et d'herbe affouragée n'ont pas été mesurées. Les pesées des génisses ont été bimensuelles jusqu'à l'âge de 18 mois, puis mensuelles jusqu'au premier vêlage et hebdomadaires pour les vaches. La hauteur au garrot, le tour de poitrine, la largeur aux hanches ont été mesurées chez les génisses à la fin de la période expérimentale avant la mise à l'herbe, l'année suivante avant la mise à l'herbe et avant la rentrée pour le premier vêlage.

Le lait produit par chaque vache a été pesé à chaque traite (deux par jour). Il a été échantillonné, après homogénéisation, 2 jours par semaine à chaque traite pour la détermination des taux butyreux et protéique. Les troubles sanitaires ont été relevés lors de la survenue de tout épisode pathologique.

\section{Analyses statistiques des données}

Les performances de croissance, de production, de reproduction et de santé ont été analysées (Sas, 1987) en fonction de l'année de naissance $(n=3,1986,1987$ et 1988), de la race $(n=2$, Holstein et Normande) et du lot ( $n=2, \mathrm{M}$ et H).

Les interactions entre les trois facteurs ont été introduites dans le modèle. Des interactions observées entre les lots et les races au niveau des mensurations et de la fertilité ont conduit à une analyse séparée des résultats dans chacune des races Holstein et Normande. L'interaction entre les années et les lots a été testée et écartée du modèle en l'absence de signification $(p>0,05)$.

\section{RÉSULTATS}

La période expérimentale s'est achevée à l'âge de $432 \pm 30$ jours d'âge des génisses. Au premier vêlage, l’âge moyen des 85 génisses primipares a été de $1070 \pm 54$ jours.

\section{Quantités ingérées (période expérimentale)}

Les génisses $M$ ont reçu moins de lait entier ( $274 \mathrm{~kg}$ soit environ $35 \mathrm{~kg} \mathrm{MS}$ ), d'aliment concentré (208 kg MS) et d'ensilage (143 kg MS) que les génisses $H$, soit l'équivalent de 400 UFL environ (tableau I). Elles ont consommé un peu plus de paille (59 kg MS) et probablement plus de foin et d'herbe en substitution à l'aliment concentré.

\section{Croissance}

Au cours de la période expérimentale, les génisses $\mathrm{M}$ ont eu un gain de poids vif inférieur à celui des génisses $\mathrm{H}(691$ contre $845 \mathrm{~g} /$ jour, $p<0,001$, tableau II). Les différences de croissance entre les deux lots apparaissent vers l'âge de 2 mois alors que débute le sevrage des génisses M (fig 1). Pendant la période d'affouragement de l'herbe, les génisses $M$ ont eu un excellent niveau de croissance, de sorte que l'écart de poids vif à la fin de la période expérimentale a principalement résulté du rationnement au cours du deuxième hiver.

À la fin de la période expérimentale, les mensurations des génisses $\mathbf{M}$ ont été inférieures à celles des génisses $\mathrm{H}$ de $3 \mathrm{~cm}$ pour la hauteur au garrot, de $2 \mathrm{~cm}$ pour la largeur aux hanches et 
Tableau I. Bilan de la conduite alimentaire des génisses durant la période expérimentale de la naissance à l'âge de 14-15 mois.

\begin{tabular}{|c|c|c|}
\hline & \multicolumn{2}{|c|}{ Lot } \\
\hline & Modéré-M & Haut-H \\
\hline Effectif initial & 51 & 52 \\
\hline Âge au sevrage (jours) & 66 & 97 \\
\hline \multicolumn{3}{|l|}{ Poids vif $(\mathrm{kg})$} \\
\hline Naissance & 41 & 41 \\
\hline Sevrage & 90 & 126 \\
\hline Fin de l'affouragement en vert & 240 & 266 \\
\hline Fin de la période expérimentale & 335 & 404 \\
\hline \multicolumn{3}{|l|}{ Quantités consommées } \\
\hline \multicolumn{3}{|c|}{ Naissance-fin de l'affouragement en vert ${ }^{a}$} \\
\hline Lait entier $(\mathrm{kg})$ & 366 & 640 \\
\hline Aliment concentré ( $\mathrm{kg} \mathrm{MS}$ ) & 125 & 270 \\
\hline \multicolumn{3}{|l|}{ Deuxième hiver } \\
\hline Ensilage (kg MS) & 572 & 721 \\
\hline Aliment concentré (kg MS) & 61 & 124 \\
\hline Paille (kg MS) & 64 & 5 \\
\hline $\mathrm{CMV}(\mathrm{kg})$ & 9 & 12 \\
\hline
\end{tabular}

${ }^{a}$ Les consommations de foin en hiver et d'herbe verte affouragée en été n'ont pas été mesurées.

de $12,7 \mathrm{~cm}$ pour le tour de poitrine $(p<0,001$, tableau II).

À la naissance, les génisses Holstein ont été plus légères que les génisses Normandes $(40,0$ contre $43,4 \mathrm{~kg}, p<0,02$ ) mais les génisses Holstein ont été plus lourdes à la fin de la période expérimentale. Leur gain de poids vif a été de $771 \mathrm{~g} /$ jour au lieu de $750 \mathrm{~g} / \mathrm{jour}$ pour les génisses Normandes $(p<0,004)$. Cet écart est dû aux gains de poids des 8 génisses Normandes nées en 1987 ( $728 \mathrm{~g} /$ jour) alors que le gain des 22 génisses Holstein contemporaines a été de $810 \mathrm{~g} / \mathrm{jour}$. À la fin de la période expérimentale, la totalité des génisses Holstein a été plus haute au garrot que la totalité des génisses Normandes $(+2,1 \mathrm{~cm}, p<0,02)$. En revanche, les différences ne sont pas significatives entre les génisses des races Holstein et Normande qui vêleront (tableau II).
Durant la période postexpérimentale, les génisses $\mathrm{M}$ ont eu un gain de poids vif supérieur à celui des génisses $H$ (554 contre 459 $\mathrm{g} /$ jour, $p<0,001$, tableau II). Avant le premier vêlage, les mensurations des génisses $\mathbf{M}$ et $\mathbf{H}$ ont été identiques. Cependant, au milieu de la période postexpérimentale, la largeur aux hanches est restée plus faible de $1,1 \mathrm{~cm}(p<$ 0,01 ) pour les génisses $M$, alors que la hauteur au garrot et le tour de poitrine ont été équivalents (tableau II).

La croissance compensatrice a été moins importante chez les génisses Holstein $(+79 \mathrm{~g} /$ jour, $p<0,001)$ que chez les génisses Normande $(+128 \mathrm{~g} /$ jour, $p<0,001)$. En race Holstein, la compensation (70\%) s'est produite surtout dès la première mise à l'herbe ( 100 jours, $+195 \mathrm{~g} /$ jour, $p<0,001$ ) puis pendant l'hiver suivant ( $+58 \mathrm{~g} / \mathrm{jour}$, ns) et la différence 
Tableau II. Poids vifs et gains de poids vif des génisses et des vaches.

\begin{tabular}{|c|c|c|c|c|c|c|}
\hline & $\begin{array}{c}\text { Modéré-M } \\
\text { Holstein }\end{array}$ & $\begin{array}{c}\text { Haut-H } \\
\text { Holstein }\end{array}$ & $\begin{array}{l}\text { Modéré-M } \\
\text { Normande }\end{array}$ & $\begin{array}{c}\text { Haut-H } \\
\text { Normande }\end{array}$ & $\begin{array}{c}\text { Effet } « L o t » \\
H F\end{array}$ & NO \\
\hline Âge au premier vêlage (jours) & 1069 & 1082 & 1079 & 1071 & $\mathrm{~ns}$ & ns \\
\hline Âge au deuxième vêlage (jours) & 1445 & 1445 & 1447 & 1449 & ns & $\mathrm{ns}$ \\
\hline Effectif premier/deuxième/troisième vêlage $(n)$ & $31 / 20 / 16$ & $24 / 13 / 9$ & $13 / 8 / 3$ & $17 / 6 / 4$ & & \\
\hline \multicolumn{7}{|l|}{ Gain de poids vif des génisses (g/jour) } \\
\hline Période expérimentale & 702 & 862 & 664 & 819 & 0,001 & 0,001 \\
\hline Période postexpérimentale & 543 & 464 & 581 & 441 & 0,001 & 0,001 \\
\hline De la naissance à après le premier vêlage & 544 & 571 & 550 & 556 & 0,01 & ns \\
\hline \multicolumn{7}{|l|}{ Poids vif des vaches présentes $(\mathrm{kg})$} \\
\hline Après le premier vêlage, une lactation & 662 & 658 & 636 & 641 & 0,003 & $\mathrm{~ns}$ \\
\hline Après le deuxième vêlage, deux lactations & 655 & 679 & 696 & 691 & ns & ns \\
\hline Après le troisième vêlage, trois lactations & 726 & 723 & 789 & 701 & $\mathrm{~ns}$ & 0,02 \\
\hline \multicolumn{7}{|l|}{ Hauteur au garrot $(\mathrm{cm})$} \\
\hline À la fin de la période expérimentale & 119,4 & 122,5 & 117,1 & 120,8 & 0,01 & 0,005 \\
\hline À la fin du premier été suivant & 131,4 & 132,3 & 130,3 & 129,8 & ns & ns \\
\hline À la fin du deuxième été suivant & 135,2 & 136,7 & 135,0 & 134,8 & ns & ns \\
\hline \multicolumn{7}{|l|}{ Largeur aux hanches $(\mathrm{cm})$} \\
\hline À la fin de la période expérimentale & 42.8 & 44,5 & 42,0 & 44,8 & 0,002 & 0,002 \\
\hline À la fin du premier été suivant & 51,8 & 52,7 & 51,3 & 52,5 & $\mathrm{~ns}$ & ns \\
\hline À la fin du deuxième été suivant & 56,0 & 56,4 & 55,4 & 55,5 & $\mathrm{~ns}$ & ns \\
\hline \multicolumn{7}{|l|}{ Tour de poitrine $(\mathrm{cm})$} \\
\hline À la fin de la période expérimentale & 159,3 & 173,3 & 159,0 & 170,4 & 0,001 & 0,001 \\
\hline À la fin du premier été suivant & 186,2 & 190,6 & 187,6 & 186,8 & 0,01 & ns \\
\hline À la fin du deuxième été suivant & 201,2 & 204,5 & 203,8 & 200,2 & 0,05 & ns \\
\hline
\end{tabular}

de poids vif observée vers l'âge de 6 mois s'est retrouvée à proximité du premier vêlage (fig 1). Chez les génisses de race Normande, la compensation a été importante tout au long du premier $(+300 \mathrm{~g} /$ jour, $p<0,001)$ et du deuxième pâturage $(+90 \mathrm{~g} /$ jour, $p<0,05)$ mais nulle en hiver. Les génisses Normandes $\mathrm{M}$ et $\mathrm{H}$ ont présenté le même poids vif lors de la deuxième mise à l'herbe et les génisses $M$ pèsent $20 \mathrm{~kg}$ de plus vers l'âge de 1000 jours. La croissance compensatrice des génisses Normandes $\mathrm{M}$ a été élevée dans la seconde moitié des périodes de pâturage (été et automne) comparativement aux génisses Holstein. Avant le premier vêlage (tableau II), une différence significative $(p<0,05)$ a subsisté sur le tour de poitrine dans la race Holstein en faveur des génisses $H$, l'inverse est obtenu en race Normande (NS).

Après le premier vêlage, les vaches primipares Holstein $\mathrm{M}$ ont été plus légères que les génisses $\mathrm{H}$ de $36 \mathrm{~kg}(p<0,001)$. En revanche, les vaches primipares Normandes des deux traitements ont eu le même poids vif $(+5 \mathrm{~kg}$ pour les génisses $\mathrm{H}, \mathrm{NS}$ ). Les différences au sein des deux races se maintiennent tout au long de la première lactation (fig 3).

Les 32 vaches ( 25 Holstein et 7 Normandes) qui ont eu trois vêlages pesaient $640 \mathrm{~kg}$ après le premier, $673 \mathrm{~kg}$ après le second et $728 \mathrm{~kg}$ après le troisième. En race Holstein, ces poids 
ont été de 638,667 et $725 \mathrm{~kg}$ respectivement (tableau II).

\section{Reproduction}

Quatre-vingt-dix neuf génisses ont été mises à la reproduction (tableau III). Les génisses des deux races avaient en moyenne le même âge. À la première insémination, les génisses Holstein $\mathrm{M}$ pesaient $23 \mathrm{~kg}$ de moins que les génisses $\mathrm{H}$ $(p<0,02)$. L'écart était plus faible dans la race Normande $(9 \mathrm{~kg})$.

Après la première insémination, la fertilité des génisses Holstein a été satisfaisante dans le lot $\mathrm{M}$ mais faible dans le lot $\mathrm{H}(77,1$ contre $47,1 \%, p<0,01)$. Après deux inséminations et globalement, l'écart de fertilité se réduit à 24,2 $(p<0,02)$ et $17,9(p<0,05)$ points respectivement. La fertilité des génisses Holstein a été inférieure à celle des Normandes : 76,8 \% au lieu de $100 \%$ après deux inséminations (tableau III). Les génisses Holstein ont eu des gestations simples plus courtes de 6 jours par comparaison aux génisses Normandes $(p<0,001)$ et elles ont eu plus de gestations gémellaires ( 4 contre 0 ).

Les génisses Normandes $\mathrm{M}$ et $\mathrm{H}$ ont eu une fertilité identique. Au total, 87 génisses ont débuté une gestation. Dans les deux races, les traitements alimentaires n'ont pas eu d'effets sur la durée de la gestation, les conditions de vêlage et le poids ainsi que la vitalité des veaux à la naissance.

Les poids des veaux à la naissance ont été élevés $(44,1 \mathrm{~kg} \pm 3,7$ pour les mâles et $39,5 \pm$ $4,9 \mathrm{~kg}$ pour les femelles). Cependant, le ratio «poids du veau/poids de la mère après le vêlage " a été faible $(6,99 \% \pm 0,76$ pour les mâles et $6,11 \% \pm 0,87$ pour les femelles). Ce ratio a été plus élevé avec les vaches Holstein primipares $\mathrm{M}$ car elles sont plus légères.
Fig 1. Effet du traitement alimentaire et de la race sur l'évolution du poids vif des génisses vêlées.

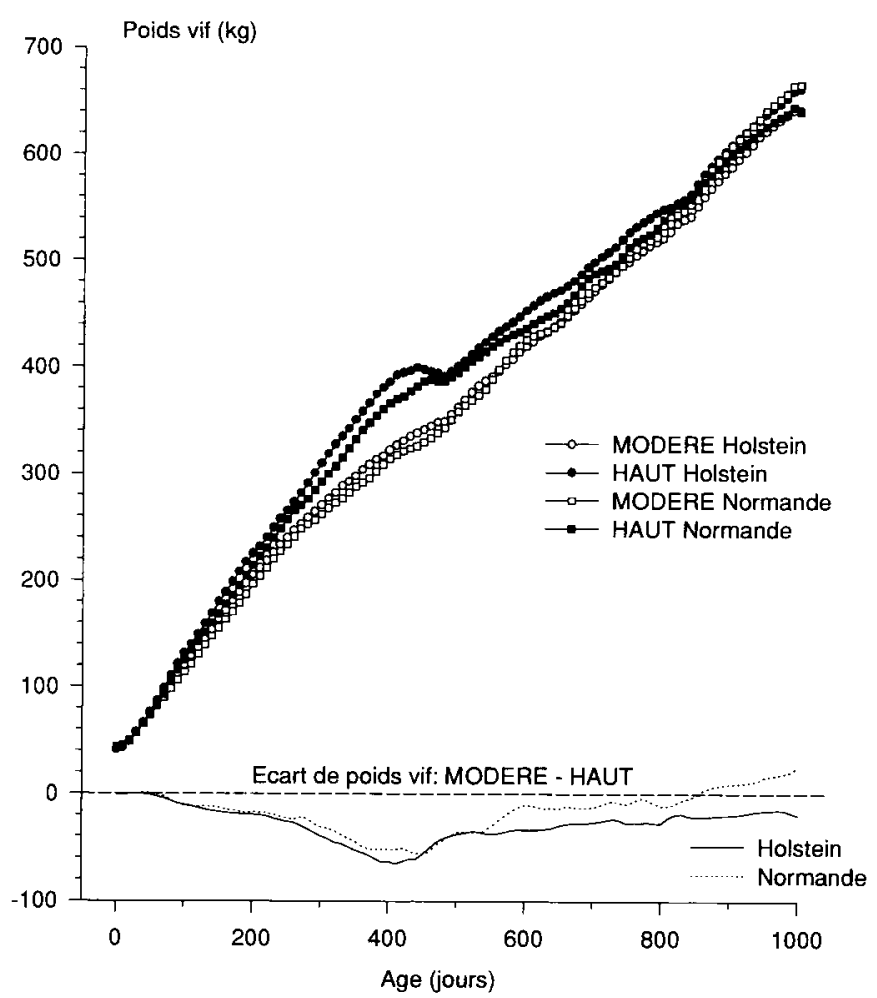




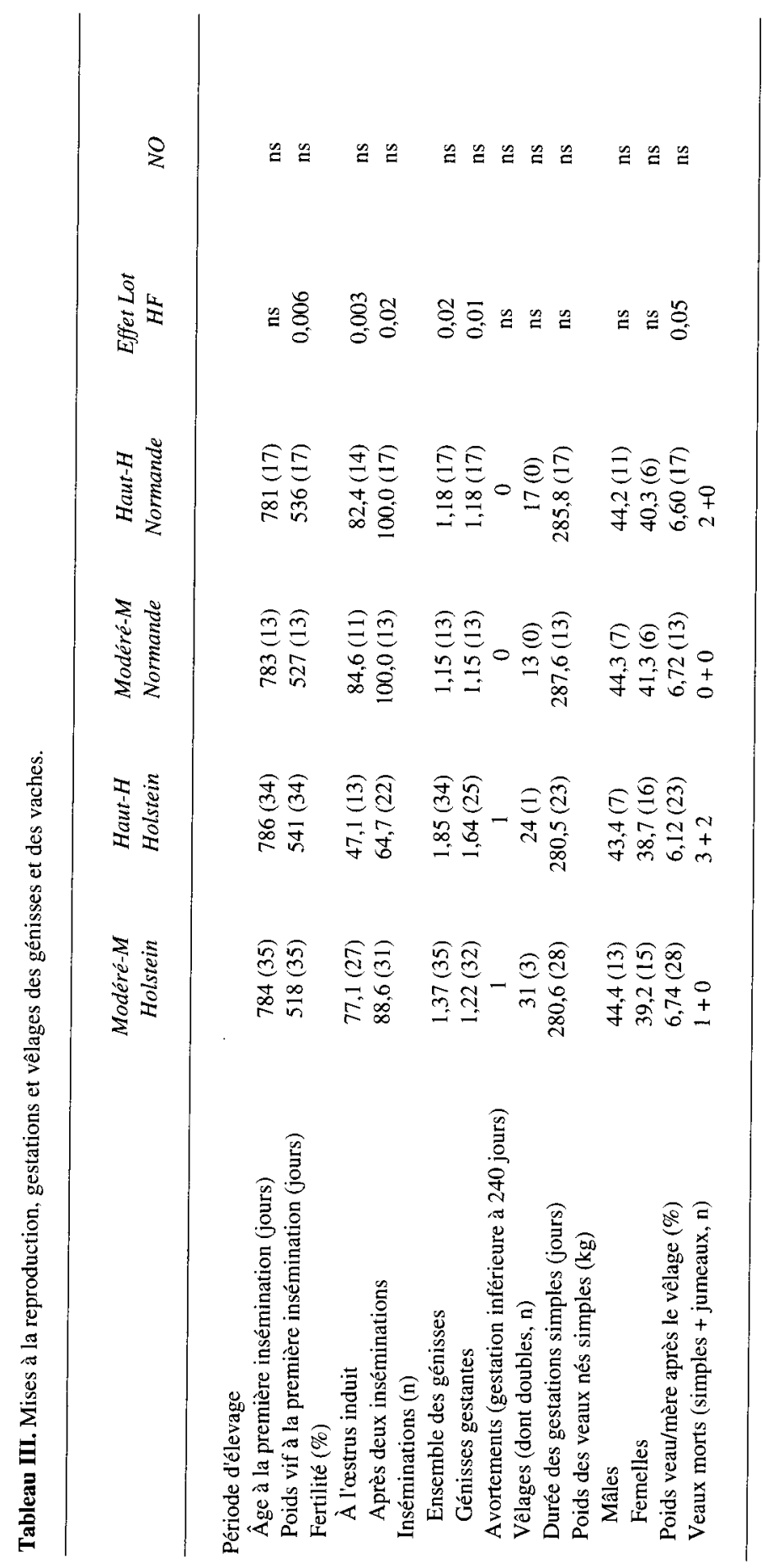


Effet du niveau d'alimentation énergétique de génisses laitières

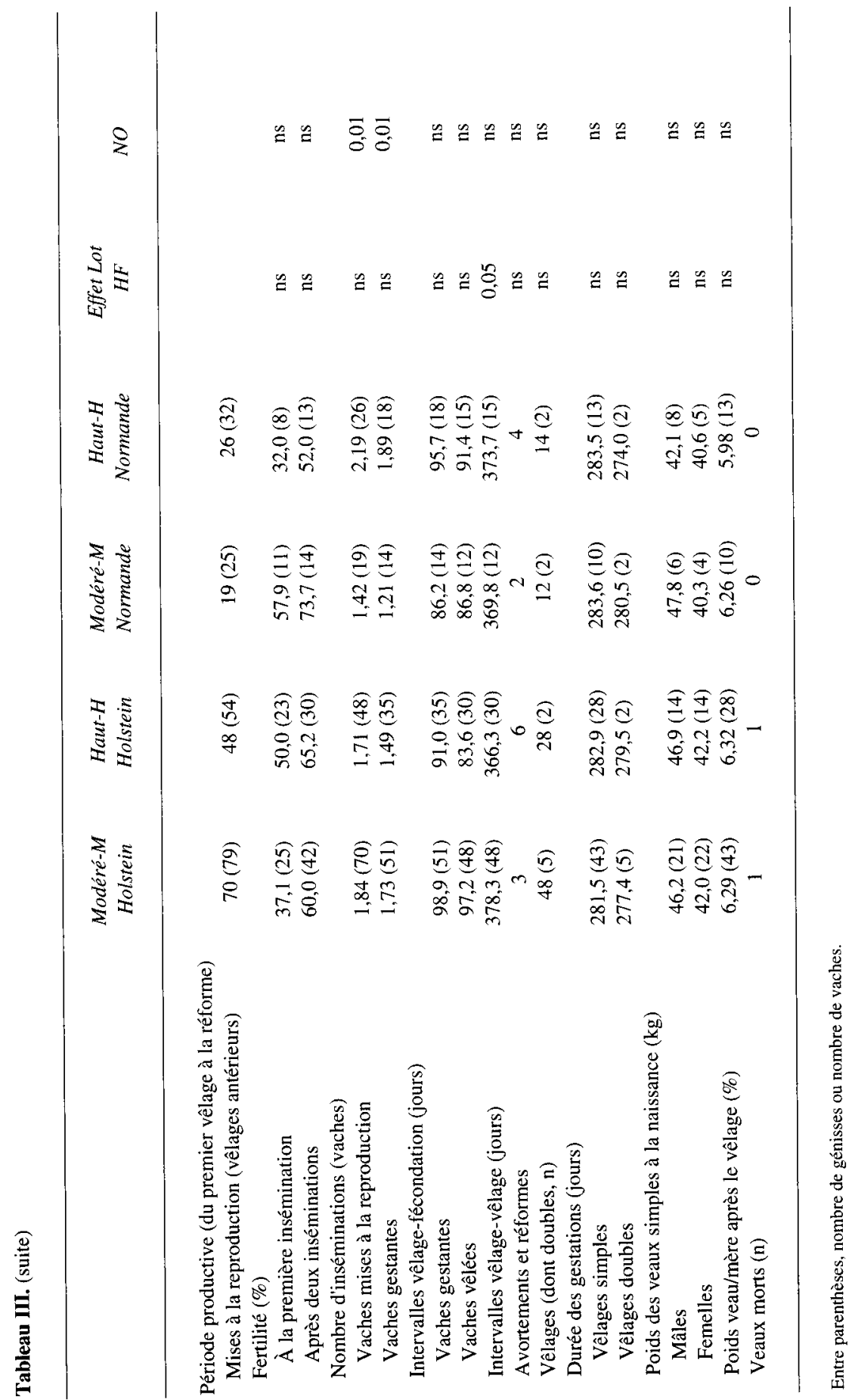


Quatre-vingt-dix pour cent des vaches Holstein ayant vêlées ont été mises à la reproduction contre $79 \%$ des vaches Normandes (tableau III). La fertilité des vaches Holstein $\mathrm{M}$ ou $\mathrm{H}$ a été identique. Cependant, les vaches Holstein $\mathrm{M}$ ont un intervalle entre les vêlages successifs allongé de 12 jours $(p<0,05)$ par rapport aux vaches $H$. En revanche, les vaches Normandes $M$ sont plus fertiles que les vaches $\mathrm{H}(p<0,02)$. Les traitements alimentaires n'ont pas eu d'autres effets significatifs sur l'intervalle vêlage-insémination fécondante, la durée de la gestation, la difficulté du vêlage, le poids et la vitalité des veaux à la naissance.

\section{Lait et composition du lait}

Quatre-vingt-quatre vaches primipares ont été traites (deux avortements et un accident au premier vêlage). Quinze vaches primipares (dix Holstein et cinq Normandes) et 15 vaches multipares (sept Holstein et huit Normandes) ont eu des lactations inférieures à 20 semaines (tableau IV, fig 2). Les productions (en 20 semaines) de lait et de matières grasses et protéiques des vaches traites au moins 20 semaines n'ont pas été significativement différentes entre les vaches $M$ et $\mathrm{H}$ qu'elles soient Holstein ou Normandes, primipares ou multipares. Les taux butyreux et protéiques des vaches $\mathrm{M}$ et $\mathrm{H}$ ont été équivalents.

Dans cet essai, toutes les génisses nées durant les trois années successives ont été conservées pour l'élevage. Il en a résulté un taux excessif de renouvellement qui a conduit à une réforme rapide des vaches peu productives après l'obtention de leur référence en production laitière. Par ailleurs, la prophylaxie de la leucose a provoqué la réforme de neuf vaches Holstein et une vache Normande. Ainsi, le nombre moyen de lactations débutées par vache a été faible : 2,4 en race Holstein et 1,9 en race Normande.

Les vaches primipares Holstein ont produit plus de lait et de matières grasses et protéiques que les vaches de race Normande. Les vaches Holstein ont eu un lait plus riche en matières grasses ( $+1 \mathrm{~g} / \mathrm{kg}$ environ, NS) et plus pauvre en matières protéiques $(-2 \mathrm{~g} / \mathrm{kg}$ environ, $p<0,001)$ que les vaches Normandes.

\section{Santé et réformes}

Les problèmes sanitaires répertoriés sur les vaches primipares ou multipares ne diffèrent pas entre les lots $\mathrm{M}$ et $\mathrm{H}$ (tableau V). Les causes de réforme des vaches sont équivalentes entre les deux lots. L'infertilité a entraîné la réforme de 29 vaches (tableau $\mathrm{V}$ ).

La fréquence des troubles sanitaires et les causes de réforme n'ont pas été significativement affectées par la race. Au total, 21 des 85 premiers vêlages ont occasionné des problèmes uro-génitaux contre 5 sur 102 vêlages ultérieurs $(p<0,01)$.

Dix-huit génisses ont été réformées (tableau V). Le poids moyen des carcasses des 12 génisses Holstein mises à la reproduction mais vides a été de $290 \mathrm{~kg}$ avec un rendement de $48,1 \%$ à l'âge moyen de 1007 jours. Les carcasses commercialisées des vaches $\mathrm{M}$ et $\mathrm{H}$ ( $n=80$ ) ont eu des poids identiques : $306 \mathrm{~kg}$ avec un rendement de $46,2 \%$ pour les 35 vaches primipares et $307 \mathrm{~kg}$ avec un rendement de 47,0\% pour les 45 vaches multipares. Les carcasses des vaches Holstein $M$ ont été plus légères que celles de leurs homologues $H$ (NS). Les 33 vaches Holstein multipares ont produit des carcasses plus légères $(300,7$ contre $326,2 \mathrm{~kg}$, $p<0,05)$ avec un rendement plus faible $(43,1$ contre $47,3 \%, p<0,001$ ) que les 12 vaches Normandes.

\section{DISCUSSION ET CONCLUSION}

Dans les conditions de cet essai, une alimentation réduite en lait entier, en aliment concentré et en ensilage (soit l'équivalent de 400 UFL environ) des génisses de la naissance à l'âge de 14-15 mois a diminué leur gain de poids vif à $691 \mathrm{~g} / \mathrm{j}$ our au lieu de $845 \mathrm{~g} / \mathrm{j}$ our. Le développement pondéral a été réduit de $17 \%(69 \mathrm{~kg}$ de poids vif). En proportion, la réduction pondérale est inférieure à la réduction énergétique : la dépense énergétique augmente avec le gain 
de poids vif par suite d'un dépôt adipeux plus important (Hansson et al, 1967). Les génisses Holstein ont un croît durant le jeune âge plus élevé que les génisses Normandes (Troccon et al, 1993) résultant d'une aptitude plus élevée ou d'une plus grande agressivité facilitant une présence plus longue à l'auge (Loisel et al, 1981).

Dès le début de la période postexpérimentale, les génisses $\mathrm{M}$ ont montré leur aptitude à la croissance compensatrice au pâturage conformément aux observations antérieures (Ryan, 1990). Cependant, cette aptitude est moindre en race Holstein qu'en race Normande. En race Holstein, l'écart pondéral entre les animaux $\mathrm{M}$ et $\mathrm{H}$ diminue progressivement jusqu' au troisième vêlage. Cependant, les vaches M pourraient produire des carcasses plus légères (Troccon, 1993). En race Normande, le même écart pondéral à

Tableau IV. Production et composition du lait des vaches primipares et multipares.

\begin{tabular}{|c|c|c|c|c|}
\hline & $\begin{array}{c}\text { Modéré-M } \\
\text { Holstein }\end{array}$ & $\begin{array}{l}\text { Haut-H } \\
\text { Holstein }\end{array}$ & $\begin{array}{l}\text { Modéré-M } \\
\text { Normande }\end{array}$ & $\begin{array}{c}\text { Haut }-M \\
\text { Normande }\end{array}$ \\
\hline Premières lactations (n) & 31 & 24 & 13 & $* 16$ \\
\hline Durée (jours) & 260 & 235 & 220 & 224 \\
\hline Production maximale $(\mathrm{kg})$ & 29,1 & 29,0 & 22,7 & 21,3 \\
\hline Lait total $(\mathrm{kg})$ & 5867 & 5297 & 4026 & 3962 \\
\hline \multicolumn{5}{|l|}{ Semaines 1 à 20 de la lactation } \\
\hline Effectif & 27 & 18 & 10 & 13 \\
\hline Production initiale $(\mathrm{kg})$ & 19.8 & 21,6 & 15,8 & 15,6 \\
\hline Production maximale $(\mathrm{kg})$ & 30,6 & 31,1 & 23,6 & 22,1 \\
\hline Lait $(\mathrm{kg})$ & 3780 & 3813 & 2957 & 2784 \\
\hline Taux butyreux $(\mathrm{g} / \mathrm{kg})$ & 42,3 & 43,7 & 41,4 & 40,3 \\
\hline Taux protéique $(\mathrm{g} / \mathrm{kg})$ & 29,4 & 29,9 & 32,3 & 32,7 \\
\hline Lactations 2 et suivantes ( $n$ ) & 48 & 30 & 12 & 15 \\
\hline Durée (jours) & 271 & 275 & 203 & 247 \\
\hline Production maximale $(\mathrm{kg})$ & 41,9 & 41,4 & 29,5 & 29,6 \\
\hline Lait total $(\mathrm{kg})$ & 7649 & 7733 & 4098 & 5376 \\
\hline \multicolumn{5}{|l|}{ Semaines 1 à 20 de la lactation } \\
\hline Effectif & 43 & 28 & 7 & 12 \\
\hline Production initiale $(\mathrm{kg})$ & 30,9 & 30,3 & 25,5 & 23,5 \\
\hline Production maximale $(\mathrm{kg})$ & 42,1 & 41,1 & 29,5 & 30,5 \\
\hline Lait $(\mathrm{kg})$ & 4993 & 4896 & 3496 & 3699 \\
\hline Taux butyreux (g/kg) & 42,0 & 42,8 & 40,3 & 38,5 \\
\hline Taux protéique $(\mathrm{g} / \mathrm{kg})$ & 29,1 & 29,7 & 32,1 & 31,0 \\
\hline \multicolumn{5}{|l|}{ Carrière } \\
\hline Lait (kg) & 17666 & 14960 & 7776 & 8997 \\
\hline Taux butyreux (g/kg) & 40,8 & 41,9 & 41,4 & 39,5 \\
\hline Taux protéique $(\mathrm{g} / \mathrm{kg})$ & 30,2 & 30,9 & 32,5 & 33,0 \\
\hline Nombre moyen de lactations & 2,6 & 2,2 & 1,9 & 1,9 \\
\hline Durée de vie (jours) & 1910 & 1801 & 1648 & 1667 \\
\hline Durée de vie productive (jours) & 853 & 719 & 571 & 592 \\
\hline Durée de lactation (jours) & 680 & 579 & 407 & 455 \\
\hline
\end{tabular}


14 15 mois est en grande partie compensé dès le premier pâturage. Le développement squelettique suit une tendance identique. La compensation plus lente sur la largeur aux hanches tient au développement plus tardif des parties postérieures du corps lorsqu'il y a eu une restriction alimentaire précoce (Hansson et al, 1967).

La fertilité des génisses Holstein $\mathrm{M}$ a été meilleure alors que la période expérimentale était terminée depuis 1 an et que les régimes étaient identiques pour toutes les génisses. L'écart de poids vif entre les deux lots était notablement réduit $(18 \mathrm{~kg})$ mais significatif. Le moindre état d'engraissement des génisses $\mathrm{M} \mathrm{a}$ pu améliorer leur fertilité (Troccon, 1993). La fertilité identique des génisses Normandes $M$ et $\mathrm{H}$ tient sans doute à un moindre état d'engraissement dans cette race par rapport à la race Hol- stein (Dauzias, communication personnelle) et peut-être à leur croissance moindre limitant cet embonpoint. Les vaches Holstein $\mathrm{M}$ et les vaches Normandes $\mathrm{H}$ ont été moins fertiles que leurs homologues. En race Holstein, l'intervalle entre deux vêlages successifs est accru de 12 jours $(p<0,05)$. En race Normande, le nombre d'inséminations est plus faible. Les vaches Holstein $M$ sont plus légères et probablement moins grasses compte tenu des niveaux identiques de production laitière : la période d'anœstrus est prolongée et leur fécondité retardée. En revanche, les vaches Normandes $\mathrm{H}$ en particulier reprennent du poids vif dès le vêlage parce que leur niveau de production laitière est faible et le niveau d'apport en aliment concentré excessif. Leur bilan énergétique très positif pourrait favoriser la mortalité embryonnaire (Ducker et al, 1985).

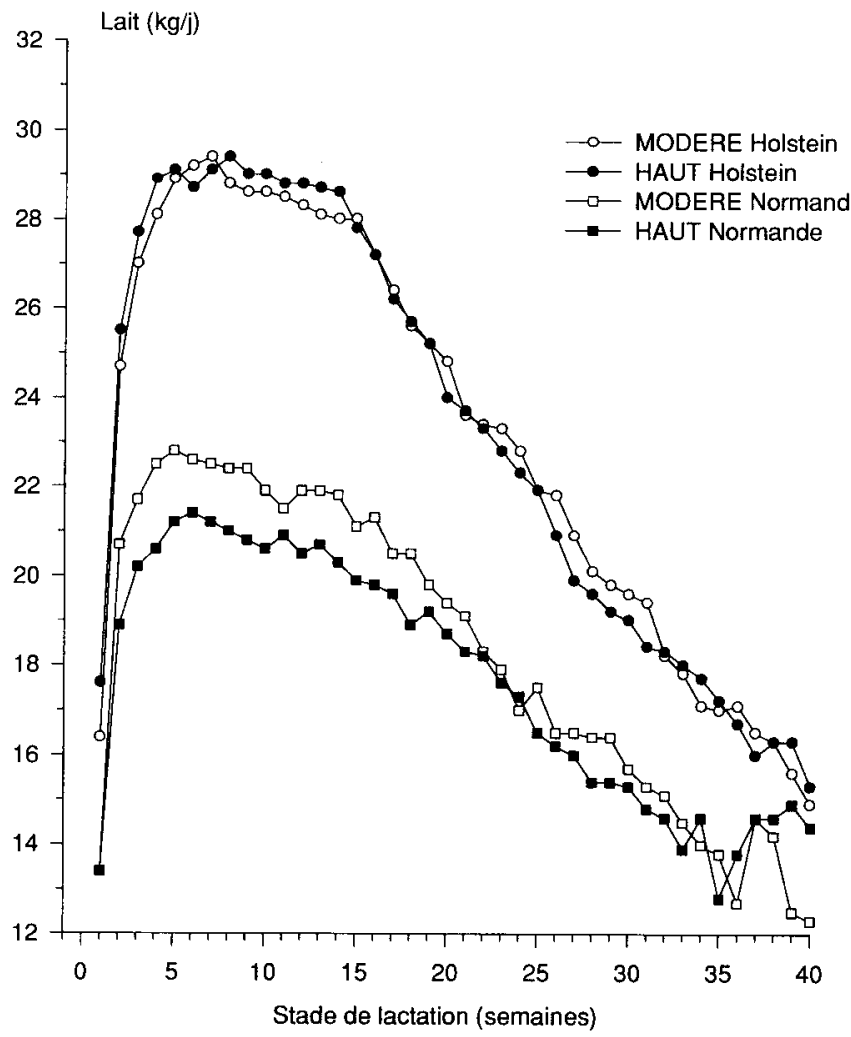

Fig 2. Effet du traitement alimentaire et de la race sur la production laitière des vaches primipares traites pendant 20 semaines au moins. 
La production laitière des vaches n'a pas été différente entre les deux traitements. Les écarts de gain de poids vif ont été créés tardivement (10 à 15 mois d'âge) et probablement en période postpubère. En effet, la production laitière des vaches est essentiellement réduite par des gains de poids vif prépubères élevés des génisses (Troccon et Petit, 1989 ; Sejrsen, 1994). Par ailleurs, la croissance compensatrice postexpérimentale des génisses $M$ a pu stimuler la différentiation et l'activité de leur glande mammaire (Park et al, 1989).

Dans cet essai, la longévité des vaches a été limitée parce que toutes les génisses ont été inséminées puis soumises à un taux de réforme fort durant la période de production. L'effectif réduit dans la race Normande (un pour deux Holstein) a pu nuire à la qualité de la répartition des génisses dans les deux lots. Par ailleurs, les écarts pondéraux ont été créés tardivement (deuxième hiver), ce qui a pu favoriser la croissance compensatrice des génisses. En effet, elle est plus limitée lorsque les rationnements sont précoces, dès la naissance ou peu après (Ryan, 1990).

Ainsi, le rationnement dans le jeune âge des génisses destinées à un vêlage tardif (30-36 mois) est zootechniquement possible car les génisses puis les vaches réalisent une croissance compensatrice. Cependant, les génisses Holstein ne devraient pas être restreintes sévèrement dans le jeune âge (4 à 6 premiers mois) pour un déve-
Fig 3. Effet du traitement alimentaire et de la race sur l'évolution du poids vif des vaches primipares traites pendant 20 semaines au moins.

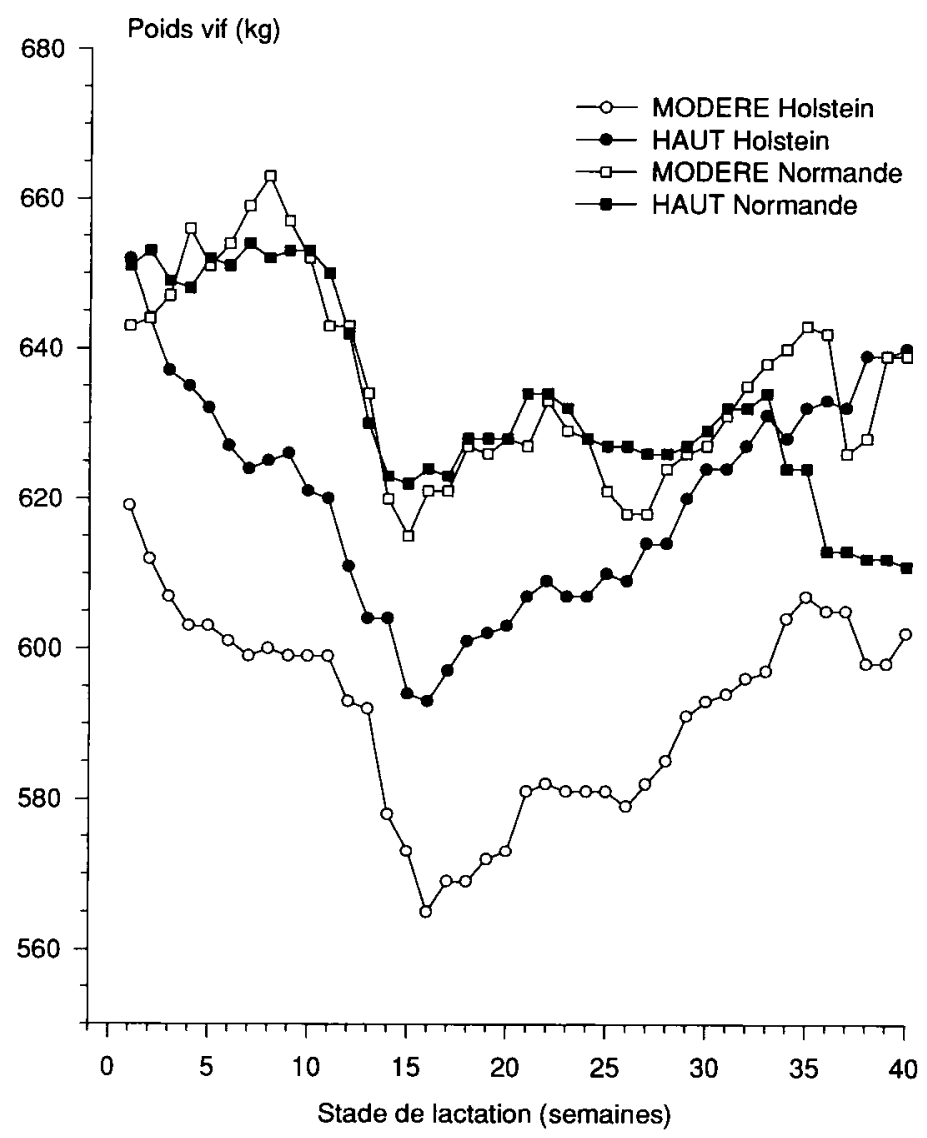


loppement corporel complet. En revanche, le rationnement énergétique ultérieur de ces génisses Holstein est indispensable pour assurer une bonne fertilité vers l'âge de 26 mois d'autant que ces génisses sont pubères précocement (Troccon et Petit, 1989).

L'économie alimentaire porte sur des aliments chers : aliment d'allaitement, aliment concentré et ensilages. Malgré leur sevrage plus précoce ( 9 semaines au lieu de 14), les génisses $\mathrm{M}$ ont reçu moins de $200 \mathrm{~kg}$ d'aliment concentré. La croissance compensatrice est réalisée au pâturage. Le croît y est efficace et peu coûteux. Cependant, une croissance compensatrice éle- vée nécessite la mise à disposition des génisses d'une herbe de qualité en quantité importante, conditions qui sont le plus souvent réunies au printemps au domaine Inra du Pin au Haras et dans l'Ouest laitier de la France.

\section{REMERCIEMENTS}

Les auteurs remercient A Bouttier (Inra du Pin au Haras) pour son assistance dans le traitement informatique des données et l'équipe des techniciens animaliers pour l'alimentation et les soins aux génisses et aux vaches.

Tableau V. Accidents sanitaires et réformes : lactations atteintes et événements recensés.

\begin{tabular}{|c|c|c|c|c|}
\hline & $\begin{array}{c}\text { Modéré-M } \\
\text { Holstein }\end{array}$ & $\begin{array}{l}\text { Haut-H } \\
\text { Holstein }\end{array}$ & $\begin{array}{l}\text { Modéré-M } \\
\text { Normande }\end{array}$ & $\begin{array}{c}\text { Haut-H } \\
\text { Normande }\end{array}$ \\
\hline Nombre de vaches (lactations) & $31(79)$ & $24(54)$ & $13(25)$ & $17(31)$ \\
\hline \multicolumn{5}{|l|}{ Affections sanitaires a } \\
\hline Poumons & $2(2)$ & $0(0)$ & $0(0)$ & $0(0)$ \\
\hline Tube digestif & $8(8)$ & $3(3)$ & $1(1)$ & $5(5)$ \\
\hline Membres & $4(4)$ & $3(3)$ & $5(7)$ & $2(2)$ \\
\hline Appareil uro-génital & $10(10)$ & $9(10)$ & $5(5)$ & $2(2)$ \\
\hline Mamelle et lactation & $42(63)$ & $29(46)$ & $14(24)$ & $14(22)$ \\
\hline Mammites & $34(49)$ & $25(37)$ & $13(23)$ & $11(18)$ \\
\hline Autres & $14(14)$ & $9(9)$ & $1(1)$ & $4(4)$ \\
\hline \multicolumn{5}{|l|}{ Causes de réformes ${ }^{b}$} \\
\hline Reproduction & $15(2,4)$ & $7(1,7)$ & $3(1,3)$ & $6(2,2)$ \\
\hline Maladie & $5(1,6)$ & $9(2,9)$ & $2(2,0)$ & $2(2,0)$ \\
\hline Niveau de production & $6(2,5)$ & $6(2,0)$ & $5(2,2)$ & $6(1,2)$ \\
\hline Accident & $5(4,0)$ & $2(1,0)$ & $3(1,7)$ & $3(2,7)$ \\
\hline \multicolumn{5}{|l|}{ Poids des carcasses commercialisées (kg) } \\
\hline Génisses ${ }^{c}$ & $274,5(5)$ & $288,0(11)$ & $213,0(2)$ & $-(0)$ \\
\hline Vaches primipares $c$ & $302,3(10)$ & $308,0(11)$ & $285,9(4)$ & $315,7(10)$ \\
\hline Vaches multipares $c$ & $295,5(20)$ & $309,3(13)$ & $327,6(7)$ & $324,2(5)$ \\
\hline Vaches mortes ou saisies à l'abattage $b$ & $1(1,0)$ & 0 & $2(1,5)$ & $2(2,5)$ \\
\hline
\end{tabular}

Aucune différence n'est significative entre les lots dans chaque race. a Les $n$ lactations affectées par les accidents sanitaires figurent dans les colonnes et les $m$ accidents recensés sont entre parenthèses; b les $n$ vaches réformées ou non, mortes, saisies figurent dans les colonnes et les rangs moyens de lactation sont entre parenthèses ; ${ }^{c}$ les poids de carcasses figurent dans les colonnes et les effectifs sont entre parenthèses. 


\section{RÉFÉRENCES}

Ducker MJ, Haggett RA, Fisher WJ, Morant SV, Bloomfield GA (1985) Nutrition and reproductive performance of dairy cattle. 1 . The effect of level of feeding on the reproductive performance of first lactation dairy heifers. Anim Prod 41, 1-12

Foldager J, Sejrsen K (1991) Rearing intensity in dairy heifers and the effect on subsequent milk production. Beretning nr 693 fra Statens Husdyrbrugsforsog, Copenhagen, $131 \mathrm{p}$

Hansson A, Brannang E, Liljedahl LE (1967) Studies on monozygous cattle twins. XIX. The interaction of heredity and intensity of rearing with regard to growth and milk yield in dairy cattle. Lantbr Högsk Ann 33, 643-649

Hoden A, Coulon JB, Faverdin P (1988) Alimentation des vaches laitières. In : Alimentation des bovins, ovins et caprins (R Jarrige, ed), Inra Publ, France, 135-158

Jammes H, Djiane J (1988) Le développement de la glande mammaire et son contrôle hormonal dans l'espèce bovine. Inra Prod Anim I, 299-310

Loisel J, Bois M, Clavreul JC (1981) Comportement des génisses de race Normande élevées en lots avec des génisses de race Pie-Noir. EDE Mayenne, $12 \mathrm{p}$

Park CS, Baik MG, Keller WL, Berg IE, Erickson GM (1989) Role of compensatory growth in lactation: a stair step regime modulates differentia- tion and lactation of bovine mammary gland. Growth, Development \& Aging 53, 159-166

Pelot J, de Fontaubert Y, Chupin D, Terqui M (1984) Management of reproduction in cattle: ovarian activity, hormonal treatments and fertility. In : The Reproductive Potential of Cattle and Sheep (Inra Publ, ed), Les Colloques de l'Inra 27, 55-70

Robelin J (1986) Bases physiologiques de la production de viande bovine : croissance et développement des bovins. In : Production de viande bovine (D Micol, ed), Inra, Paris, 35-60

Ryan WJ (1990) Compensatory growth in cattle and sheep. Nut Abstr Rev 60, 653-664

Sejrsen K (1994) Relationships between nutrition, puberty and mammary development in cattle. Proc Nutr Soc 83, 103-111

Statistical Application Systems (1987) Sas/Stat Guide for Personal Computers, (Version 6). Sas Institute Inc, Cary, NC, États-Unis, 1028 p

Troccon JL (1993) Effects of winter feeding during the rearing period on performance and longevity in dairy cattle. Livest Prod 36, 157-176

Troccon JL, Daburon E, Gallard Y, Muller A (1993) Systèmes d'élevage des génisses laitières. Comparaison des races Holstein et Normandes. Inra Prod Anim 6, 277-288

Troccon JL, Petit M (1989) Croissance des génisses de renouvellement et performances ultérieures. Inra Prod Anim 2, 55-64 